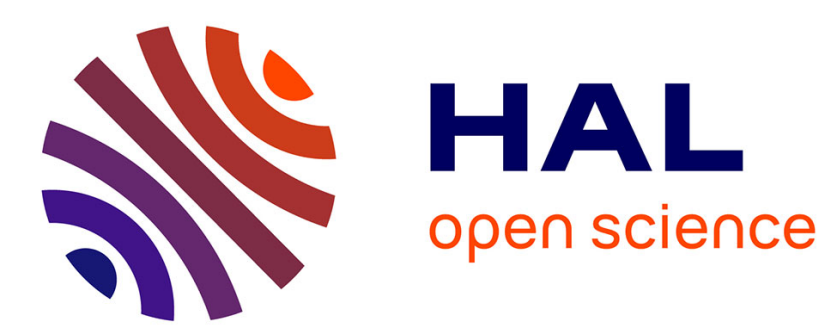

\title{
De l'utilité de l'impôt pour freiner l'effet de levier du " hors-bilan " des banques
}

Jean-Paul Nicolaï, Alain Trannoy

\section{To cite this version:}

Jean-Paul Nicolaï, Alain Trannoy. De l'utilité de l'impôt pour freiner l'effet de levier du " horsbilan " des banques. Revue d'économie financière, 2018, 131 (3), pp.151. 10.3917/ecofi.131.0151 . hal-01994827

\section{HAL Id: hal-01994827 https://hal-amu.archives-ouvertes.fr/hal-01994827}

Submitted on 27 Jul 2021

HAL is a multi-disciplinary open access archive for the deposit and dissemination of scientific research documents, whether they are published or not. The documents may come from teaching and research institutions in France or abroad, or from public or private research centers.
L'archive ouverte pluridisciplinaire HAL, est destinée au dépôt et à la diffusion de documents scientifiques de niveau recherche, publiés ou non, émanant des établissements d'enseignement et de recherche français ou étrangers, des laboratoires publics ou privés. 


\title{
DE L'UTILITÉ DE L'IMPÔT POUR FREINER L'EFFET DE LEVIER DU «HORS-BILAN » DES BANQUES
}

\author{
JeAn-Paul NiCOLAÏ* \\ ALAIN TRANNOY**
}

L

es analyses, les propositions et parfois les décisions concernant la fiscalité dans le secteur bancaire depuis la crise de 2007 relèvent de considérations très disparates : punitives, contributives, incitatives, prudentielles ou correctrices. Punitives car les banques sont considérées coupables d'excès ayant coûté très cher à la collectivité (taxe sur les primes en France et au Royaume-Uni, par exemple) ; contributives car le secteur financier présente de larges assiettes susceptibles, avec de faibles taux, de rapporter des montants importants aux Trésors publics (taxe sur les transactions financières, par exemple); incitatives, voire prudentielles, car les prises de risque inconsidérées ou les distributions de salaires astronomiques sont jugées néfastes pour la collectivité, voire pour les banques elles-mêmes ou encore dans une logique de risque systémique (taxe "systémique» mise en place en 2011) ; enfin, correctrices, car il semble bien que l'organisation bancaire offre des rendements d'échelle qui conduisent à des rentes.

Les banques, comme quelques autres secteurs, présentent une fiscalité ne relevant pas du droit commun : elles ne sont pas assujetties à la TVA, mais à une taxe sur les salaires. Cette dernière disposition est

\footnotetext{
* Chef économiste, directeur du Programme évaluation, Secrétariat général pour l'investissement. Contact : jean-paul.nicolai @ outlook.com.

** Directeur d'études, EHESS ; professeur, École d'économie d'Aix-Marseille. Contact : alain.trannoy@ univ-amu.fr.

Cet article est une version abrégée et remaniée d'un document de travail du Conseil d'analyse stratégique (aujourd'hui France Stratégie), n 2013-1.
} 
parfois contestée. L'analyse empirique ${ }^{1}$ laisse pourtant penser qu'elle impacte réellement les comportements du secteur en matière de salaire.

Les salaires ne sont pas les seuls sujets de préoccupation. Nombreux sont ceux qui considèrent que l'ensemble des activités bancaires ne fonctionnent pas efficacement et qu'une taxation de nature " correctrice " serait nécessaire. Le travail de réglementation débuté en pleine crise vise à contraindre et orienter ces activités bancaires. La séparation entre banques de détail et banques de financement et d'investissement n'aura finalement pas lieu en France, l'option retenue ayant consisté pour l'essentiel à mieux séparer les activités pour compte propre et celles à destination de la clientèle. En Europe, la proposition de règlement qui envisageait une séparation entre activités de détail et de marchés des grandes banques et qui datait de 2014 (le Conseil avait pris position en faveur de ce texte en juin 2015 ; Conseil européen, 2015) a finalement été abandonnée en octobre 2017. En revanche, les premiers pas pour l'Union bancaire ont été faits (même si le pas ultime reste à faire ${ }^{2}$ ). Surtout le Comité de Bâle a multiplié les textes, sources d'évolutions susceptibles de mieux maîtriser l'activité bancaire (accroissement des fonds propres réglementaires, mise en place d'un ratio de levier sur le bilan et le hors-bilan ${ }^{3}$, gestion du risque de liquidité, etc.). Certes, les débats sur ce qu'il est convenu d'appeler Bâle IV pourraient correspondre à une forme de marche arrière, mais les doutes sur le fonctionnement du système bancaire restent présents. Encore récemment (octobre 2018), le Comité de Bâle s'est insurgé contre les pratiques des banques qui, semble-t-il, habillent leurs bilans chaque fin de trimestre pour que la " photo » de la mesure des risques présente un visage moins risquét. Derrière ces sujets, la question de la valeur ajoutée du système bancaire - pour le moins dans ses activités les plus spéculatives - est explicitement posée, accompagnée le plus souvent en réponse d'un jugement expéditif.

Cet article se propose de mieux articuler le lien entre l'activité traditionnelle du système bancaire et la finance de marché. On y montre que cette dernière que, pour simplifier, on se propose d'assimiler à une activité d'intermédiation n'est qu'un levier de l'activité traditionnelle de « transformation » dans son rôle d' " accroissement des possibles ".

Avec ce prisme, on étudie les spécificités des métiers financiers. Il nous est alors possible de revenir à la question fiscale. Un tableau synthétique comparant différents types de fiscalité est finalement présenté avec les préconisations qui nous semblent adaptées aux trois principaux objectifs envisageables : diminuer les distorsions issues de rentes dans le secteur financier, atténuer le risque systémique et, finalement, maîtriser l'« accroissement des possibles". 
Nous expliquons d'abord en termes généraux ce que signifie ce troisième objectif qui constitue sans doute la partie la plus nouvelle de l'argumentaire. Puis nous détaillons le fonctionnement du système bancaire sous le prisme du hors-bilan. Nous abordons enfin la question fiscale avant de conclure.

\section{LE MARCHÉ COMME ACCROISSEMENT DE L'ENSEMBLE DES POSSIBLES}

L'apparition de places de marché a été décisive dans l'histoire du développement économique (Braudel, 1979). Quelle valeur peut-on donner à une place de marché ? Nous proposons de considérer qu'une place de marché rend possible des actions et des trajectoires individuelles et collectives qui seraient impossibles sinon. Nous nous proposons d'en donner une mesure dans le cas d'un modèle ultrasimplifié, le modèle d'une économie d'échange entre deux investisseurs, un prêteur et un emprunteur.

On peut définir la valeur d'un marché comme le fait de passer d'un ensemble des possibles de l'économie autarcique (un ensemble de trajectoires individuelles et collectives, par exemple), et donc d'un optimum collectif dans un tel univers, à un autre optimum associé à un univers "élargi » (et donc plus élevé). À cet écart, il faut bien sûr soustraire le coût économique et social de l'introduction de ce marché, pour en définir au total sa "valeur".

Aussi étonnant qu'il y paraisse, cette notion de valeur d'un marché n’a jamais été théorisée en tant que telle par l'analyse économique, en particulier néoclassique, alors que cette dernière met au cour de son analyse la notion d'échange. Bien sûr, il est établi depuis longtemps que l'échange est mutuellement bénéfique, puisqu'il est volontaire.

La question posée est de savoir si l'on peut chiffrer, en termes de gain de ressources initiales, ce gain de bien-être pour les échangistes. Prenons la boîte d'Edgeworth qui représente l'ensemble des allocations possibles entre deux échangistes d'une ressource initiale constituée d'un ensemble de titres sans risques et d'actifs risqués. On peut dire que tous les marchés financiers permettent la rencontre d'investisseurs ou d'épargnants d'institutionnels qui veulent échanger des titres de maturité ou de risque différents. À titre d'illustration, sur le schéma (infra), par exemple, la quantité de l'actif sans risque est mesurée sur l'axe des abscisses (actif 1), la quantité de l'actif risqué sur l'axe des ordonnées (actif 2).

Sur ce schéma (infra), on a représenté l'allocation d'autarcie de deux agents dans la boîte d'Edgeworth donné par le point A. Cette représentation graphique met ensemble la vision de l'agent 1 à partir de l'origine $\mathrm{O}_{1}$ et la vision de l'agent 2 à partir de l'origine $\mathrm{O}_{2}$. L'agent 1 
dispose de plus de titres 1 en abscisse et l'agent 2 détient plus de titres 2 en ordonnée. Leurs courbes d'indifférence ne sont pas tangentes en A, indiquant une possibilité d'échange mutuellement profitable. Un équilibre d'un marché concurrentiel est représenté par le point $\mathrm{E}$, point de tangence de deux courbes d'indifférence, la pente de la tangente donnant le rapport des prix qui équilibre exactement les offres et les demandes sur les marchés des deux biens. Cette tangente passe par l'allocation autarcique, indiquant bien qu'au prix d'équilibre, la valeur des paniers à l'équilibre est égale à celle en autarcie. Le point E est situé sur la courbe des contrats, lieu des tangences des courbes d'indifférence et donc des optima de Pareto.

Le gain de bien-être est représenté par le fait que les courbes d'indifférence passant par l'allocation d'équilibre E sont au-dessus de celles passant par l'allocation autarcique. Ce gain en bien-être est évident graphiquement, mais l'étape suivante est de traduire le gain en bien-être en termes de gains de ressources pour l'économie. Les ressources de l'économie sont mesurées par la longueur du segment $\mathrm{O}_{1} \mathrm{O}_{2}$ qui définit toutes les allocations proportionnelles des deux biens entre les deux agents. Un agent dispose du même pourcentage de chaque titre dans l'économie, lorsque son allocation est située sur ce rayon. Nous allons traduire le gain pour l'économie de la création de ce marché par une quantité mesurable sur ce rayon. Plus précisément, nous montrons qu'une mesure possible de la valeur du marché est représentée par la distance GF.

\section{Schéma}

La valeur d'un marché (en \% des ressources de l'économie) est donnée par GF sur $\mathrm{O}_{1} \mathrm{O}_{2}$

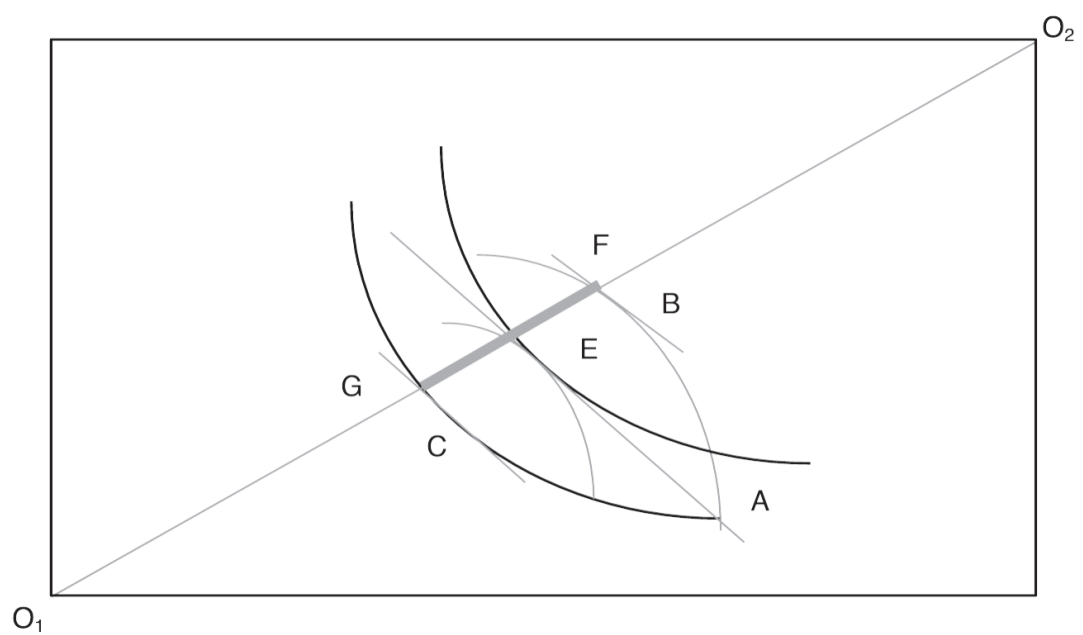

Source : d'après les auteurs. 
Comment arrivons-nous à cette conclusion ? Nous chiffrons la perte d'efficience économique que représente l'absence d'échange sur ces deux marchés. Les points $\mathrm{B}$ et $\mathrm{C}$ jouent un rôle important dans la construction de l'argument. Le point $\mathrm{C}$ donne le panier indifférent à l'allocation autarcique pour l'agent 1 qui minimise la dépense pour un vecteur de prix identique au prix d'équilibre. De même, le panier $B$ donne le panier indifférent à l'allocation autarcique pour l'agent 2 qui minimise la dépense pour un vecteur de prix identique au prix d'équilibre. Pourquoi choisir comme prix d'équilibre celui qui mesure la valeur relative d'un bien ? Tout simplement parce qu'il est la mesure de la rareté et de la désirabilité relative des deux biens dans cette économie, compte tenu des préférences des consommateurs et des ressources de l'économie. On en déduit que si l'on donne l'allocation $\mathrm{C}$ à l'agent 1 et l'allocation $\mathrm{B}$ à l'agent 2, ils sont aussi heureux que dans l'allocation de départ. Mais comme $\mathrm{C}$ et $\mathrm{B}$ ne sont pas confondus, on a l'impression que l'on économise des ressources par rapport à l'allocation initiale.

Ce n'est pas qu'une impression car si l'on projette le point $\mathrm{C}$ sur la première bissectrice (le rayon des ressources $\mathrm{O}_{1} \mathrm{O}_{2}$ ) en utilisant les prix d'équilibre, on obtient le point $\mathrm{G}$ qui est l'intersection de la droite $\mathrm{O}_{1} \mathrm{O}_{2}$ et de la droite de budget du premier agent passant par $\mathrm{C}$ et admettant pour pente le prix d'équilibre. De même le point $\mathrm{F}$ est l'intersection de la droite $\mathrm{O}_{1} \mathrm{O}_{2}$ et de la droite de budget pour le second agent avec une pente donnée par le prix d'équilibre et qui passe par le point $\mathrm{B}$. On constate immédiatement que $\mathrm{G}$ et $\mathrm{F}$ ne sont pas confondus. C'est comme si le premier agent ne consommait que les ressources $\mathrm{O}_{1} \mathrm{G}$ et le second les ressources $\mathrm{O}_{2} \mathrm{~F}$ dans l'économie autarcique. L'absence de création d'un marché entraîne donc un gaspillage de ressources mesuré par GF ou réciproquement, c'est comme si le marché apportait ces ressources supplémentaires représentées par GF. En conséquence, la valeur de la création de ce marché est représentée par le rapport de GF sur $\mathrm{O}_{1} \mathrm{O}_{2}$.

Sur l'exemple graphique, ce rapport représente entre $1 / 6$ et $1 / 5$ des ressources de l'économie, montant duquel il faut retrancher les coûts de transaction et les coûts fixes liés à la création d'un marché. Bien sûr, ce chiffre dépend entièrement des paramètres de l'économie, la plus ou moins grande rareté des biens, la préférence relative pour les différents biens synthétisée par le taux marginal de substitution et l'élasticité de substitution, ainsi que la disparité des dotations en ces différents biens pour les deux agents. Il va de soi que si l'agent qui préfere relativement plus le bien 1 est très richement doté en bien 2 , la valeur de marché sera plus forte que s'il y a congruence entre les dotations initiales et les préférences. 
Voilà une mesure de l'accroissement des possibles apporté par la création d'un marché qui est évidemment statique. Ensuite, cette création de valeur engendre une autre trajectoire pour l'économie. On peut dire que la démultiplication des marchés financiers répond à cet objectif d'exploiter le moindre gain à l'échange. Encore faut-il qu'il n'y ait pas tromperie sur la marchandise, comme dans tout marché même le plus banal.

Toute innovation radicale, de la création de produit à la mise en place de nouveaux cadres institutionnels, en passant par la pure innovation technologique, modifie par définition le champ des possibles pour une économie. La mise en place d'un système financier, par sa place au cour des échanges de tous les acteurs, est toutefois spécifiquement déterminante (Pascali, 2016), au même titre que les innovations aussi radicales que l'électricité, le moteur à explosion, la roue, le téléphone, l'Internet, etc.

\section{SPÉCIFICITÉS \\ DE L'ACTIVITÉ BANCAIRE}

Les fonctions du système bancaire peuvent être regroupées en deux grandes familles de fonction :

- l'activité de transformation, qui se ferait sans recours aux marchés financiers (dépôts transformés en crédits au logement et à l'entreprise en simplifiant), ou au travers d'achats d'obligations d'État ou d'entreprise ;

- l'activité d'intermédiation qui passe par une activité sur les marchés financiers et notamment par la vente de produits «structurés".

Si l'on retient le prisme du risque, cette séparation peut être rapprochée d'une décomposition "bilan " et " hors-bilan », le risque étant porté par la banque dans le premier cas et non dans le second (cf. encadré 1 infra). Une telle décomposition ne correspond pas à la nomenclature de la comptabilité bancaire où l'on ne raisonne pas en termes de risque.

De fait, il n'y a pas recouvrement intégral de l'intermédiation et du hors-bilan, mais ce découpage simplificateur va être utile pour penser la façon dont la taxation peut être un outil pour freiner la prise de risque excessive du secteur financier et le risque systémique, ou la prise de risque de l'économie dans son ensemble que permet le secteur financier. 


\section{Qu'entend-on par « hors-bilan ॥ ?}

1 - Le hors-bilan engage l'entreprise vis-à-vis d'un tiers ou l'inverse sans que la contrepartie de cet engagement ne se trouve au bilan pour un montant de même nature ou de même niveau.

Il peut s'agir d'engagements vis-à-vis des salariés, des fournisseurs, des clients, ou de tout autre tiers. Ainsi, tout contrat signé avec un tiers va engager l'entreprise hors-bilan. Le droit des contrats ouvre très largement la famille des éléments hors-bilan envisageables. Bien entendu, une entreprise n'a pas la même valeur, à bilan identique, si elle a signé un contrat qui l'engage ou qui engage des tiers dans le futur.

La réglementation oblige aujourd'hui les entreprises à joindre en annexe du bilan une présentation des engagements reçus ou donnés par une entreprise qui n'apparaissent pas au bilan, mais sans définir explicitement ce que sont ces engagements.

Pour les banques, la cession de prêts, les engagements de financement (lignes de crédit, autorisations de découvert pour les particuliers), les garanties données sur des prêts et les opérations sur les produits dérivés de type contractuel (swaps, futures, etc.) sont les plus souvent cités comme des éléments de hors-bilan.

2 - Les nouvelles normes internationales de comptabilité (IFRS, International Financial Reporting Standards) réintroduisent une partie des éléments hors-bilan dans le bilan pour mieux en apprécier l'impact sur la valeur de l'entreprise. Ce que nous appelons dans ce travail " hors-bilan » est l'ensemble des instruments financiers dérivés qui, dans cette norme, relèvent du bilan.

L'un des principes fondamentaux des normes comptables IFRS est d'intégrer dans le bilan d'une entreprise le maximum d'éléments qui donnent une indication sur la valeur de l'entreprise. Ces normes conduisent dès lors à intégrer en les valorisant des éléments qui auparavant relevaient du "hors-bilan ». En particulier, les instruments financiers " dérivés " sont en comptabilité IFRS valorisés dans le bilan des banques et des entreprises en général. Ces instruments entrent pour l'essentiel dans le "portefeuille de négociation » (ou de «transaction $")^{5}$.

3 - Le portefeuille de négociation est lui-même décomposé en trois types d'instruments.

Le portefeuille de négociation est aujourd'hui découpé en trois « niveaux de juste valeur": le premier niveau concerne les instruments pour lesquels la valorisation s'effectue sur la base d'un prix sur un marché organisé ; le deuxième est relatif aux instruments valorisés 
sur la base d'un modèle s'appuyant sur des données de marché ; le troisième a trait aux instruments dont la valorisation à la juste valeur nécessite un modèle et des données non directement issues de prix sur un marché organisé.

À titre d'exemple, l'une des grandes banques françaises (total de bilan supérieur à $1380 \mathrm{Md} €$ ) affichait dans ses comptes à la fin de 2016 un portefeuille de transaction (au sens élargi d'instruments à la juste valeur par le résultat) de $450 \mathrm{Md} €$ à l'actif dont $157 \mathrm{Md} €$ sous forme de dérivés. La répartition entre les trois niveaux de juste valeur donnait pour les niveaux 2 et $3: 346 \mathrm{Md} €$. Le passif à la juste valeur était de $390 \mathrm{Md} €$ environ, dont $190 \mathrm{Md} €$ de dérivés. Les niveaux 2 et 3 totalisaient au passif $375 \mathrm{Md} €$.

Comme le souligne l'encadré, les IFRS intègrent aujourd'hui ce que l'on appelait " hors-bilan » (en parlant d'instruments financiers dérivés) dans le bilan. Mais cette stratégie comptable ne rend pas compte, via le concept de "juste valeur ", de l'ensemble représenté par ces engagements. En effet, engagements et juste valeur ne coïncident pas. Un dérivé comme, par exemple, une option de vente peut " engager" pour 100 et son " prix " être proche de zéro car naturellement actualisé et prenant en compte la probabilité d'exercice vu d'aujourd'hui. Il y a donc une différence essentielle entre, d'une part, la mesure des risques et, d'autre part, celle du "levier " que l'activité financière apporte. Certes l'usage de stress tests fondés sur les justes valeurs peut rapprocher ces deux mesures des risques et des engagements, mais une vision probabilisée et actualisée réduira toujours la taille de l'engagement.

\section{Le bilan et l'activité de transformation}

Le rôle de transformation du risque apparaît assez simplement au bilan des banques. C'est le cas des crédits et des dépôts notamment. Il y a bien transfert de risque entre agents et, ici, la banque porte ce risque pour l'essentiel. C'est la mutualisation et la capacité à sélectionner ses débiteurs qui créent la valeur économique (qui accroît les possibles, par exemple, en rendant accessible le crédit à certains agents économiques) ${ }^{6}$.

La transformation ici est essentiellement une transformation de maturité et ne prend pas en considération d'autres types de transferts de risque qui pourraient naturellement y être rattachés : des garanties, des options de tout type, etc. Pour simplifier l'exposition, ces autres transferts de risque sont assimilés à la fonction d'intermédiation. 


\section{Le hors-bilan et l'intermédiation}

La banque transfere également le risque entre agents en étant un simple intermédiaire. Dans sa fonction de "transformation " décrite supra, elle structure le risque et le porte. Dans son rôle d' 'intermédiation ", elle le structure sans théoriquement le porter, ou en tout cas pas dans sa totalité.

Quelques exemples montrent où se loge le risque résiduel : un investisseur veut acheter une obligation ; la banque vend cette obligation, alors qu'elle n'en dispose pas forcément en portefeuille, dès lors qu'elle pense pouvoir se la procurer pour moins cher ; par ailleurs, elle va couvrir ce risque : soit effectivement en l'annulant par l'achat de cette obligation auprès d'un tiers ; soit en la répliquant au travers d'autres produits (un contrat dérivé, par exemple). Dans ce second cas, le service qui a vendu l'obligation va se retourner vers différents autres services en interne de la banque ou d'une autre banque. On observe dans ce cas deux choses : (1) le risque n'est pas toujours parfaitement couvert ; (2) les services qui fournissent les éléments de la couverture vont eux-mêmes se retourner vers d'autres services pour couvrir leurs positions (c'est ce mécanisme qui est à l'origine pour partie de l'« inflation des bilans" y compris, on le comprend ici, des engagements horsbilan).

Première difficulté : moins les positions sont parfaitement couvertes, plus l'espérance de gain est grande, et plus la fonction d'intermédiation de la banque, une fois "optimisée ", conduit cette dernière à porter le risque pour partie. En effet, les systèmes de mesure des risques ne peuvent pas toujours identifier totalement le risque "résiduel " ne serait-ce que parce que la modélisation retenue dans le système de risque est une approximation souvent du même ordre que l'outil qui permet de déterminer la couverture. Par ailleurs, la partie résiduelle correspond souvent à un risque aux propriétés statistiques non standards, car la partie standard est facilement transférée par la banque aux investisseurs.

Deuxième grande difficulté, la dimension temporelle. Une option d'achat vendue à un investisseur sera "gérée " dans le temps, en essayant de tirer le maximum de revenus de cette gestion tout en contraignant les risques à respecter l'engagement pris par la banque à tenir dans un "budget de risque " prédéfini. Le "produit " vendu, l'option d'achat dans cet exemple, n'est "produit " - c'est-à-dire " répliqué »- qu'une fois qu'il est cédé, et on ne saura qu'à l'échéance combien la banque a gagné ou perdu. On imagine aisément que pour des produits plus complexes qu'une option d'achat, l'aléa peut-être très élevé. De plus, à chaque position prise dans cette dynamique de 
couverture, le mécanisme de réplication de service à service, de banque en banque, démultiplie les enjeux de l'opération initiale. Il n'existe à ce jour aucun système de mesure de risque - voire de concept de risque qui permette de tracer précisément le risque lié à l'opération, pourtant simple, de vente d'une option d'achat. Pour cela, il faudrait modéliser le comportement de gestion de la couverture de tous les opérateurs qui sont intervenus en cascade, sachant que l'objectif de gestion de la couverture de chaque opérateur consiste à dégager le revenu maximal sous contrainte d'une enveloppe de risque mesurée à l'aune de l'activité du service concerné.

Il existe une ultime difficulté : le processus de production d'un produit ou d'un service financier vendu dans un pays peut nécessiter des opérations dans d'autres pays (par des entités de la même banque) et la localisation du risque et des profits n'est pas aisée. Certes les exigences en matière de prix de transfert ont entraîné une forme de clarification - et des réorganisations - au sein des banques. Toutefois, compte tenu des difficultés de mesures signalées supra, on imagine mal comment les politiques actuelles en matière de prix de transfert pourraient totalement permettre la localisation d'une opération qu'une fiscalité ou une réglementation pourraient souhaiter/nécessiter.

\section{Le levier de la finance sur l'activité traditionnelle}

Il est important de comprendre que l'activité financière "horsbilan » fait levier (en termes d'accroissement des possibles) sur l'activité traditionnelle, qu'il s'agisse de crédit à taux variables " capés » pour le crédit ou de " produits garantis » pour l'épargne, voire de produits pour la trésorerie d'entreprise. Grâce à cette activité sur les marchés, les produits d'épargne et de financement offrent des possibilités nouvelles aux agents non financiers. C'est bien sûr aussi vrai pour les activités de financements complexes, même lorsqu'il s'agit simplement de certains financements d'actifs. Lorsque ces actifs ne sont pas diversifiables au sein d'un portefeuille large du même type d'actif (bateaux, avions, infrastructures), les facteurs de risque doivent être couverts autrement, souvent grâce à l'utilisation de produits dérivés traités sur les marchés financiers.

Comme indiqué plus haut, c'est dans un sens très spécifique qu'est ici associé le hors-bilan à l'activité d'intermédiation, par opposition à l'activité traditionnelle de transformation. Toutefois cela ne modifie pas le raisonnement que nous souhaitons conduire en matière de fiscalité, et cela permet de mieux comprendre l'articulation entre transformation et intermédiation.

Ainsi il convient de remarquer que les subprimes sont "issus " de l'activité traditionnelle des banques (crédit immobilier) et que c'est 
l'activité de marché qui a apporté le « levier " (par la mutualisation des risques au sein d'un produit ensuite découpé en " tranches » de niveaux de risque). Ce levier devait permettre, certes, d'enrichir certains intermédiaires, mais aussi de rendre possible l'acquisition de leur maison par une fraction peu aisée de la population américaine, et d'apporter à certains investisseurs des supports de placements présentant - croyaient-ils - le profil rendement-risque qu'ils recherchaient.

\section{FISCALITÉ}

Dans la réflexion en matière de fiscalité, il apparaît nécessaire de séparer ce qui relève :

- de l'existence de rentes issues des modèles économiques des banques ;

- de l'excès de prise de risque bancaire, y compris systémique ;

- de l'accroissement de risque économique et social lié à l'accroissement des possibles, ou de l'apparition de possibles non désirés.

Les deux derniers objectifs peuvent apparaître connectés. Ils le sont effectivement, mais même une petite structure peut créer des possibles non désirés.

Les différents instruments fiscaux susceptibles de ponctionner un système bancaire peuvent être examinés selon l'efficacité avec laquelle ils atteignent l'un de ces trois objectifs. Notre propre vision de cette efficacité relative est résumée dans le tableau (infra) où nous avons ombré les cases les plus pertinentes pour chaque objectif. Cette partie a pour objet de proposer une argumentation en appui de celui-ci.

\section{La rente}

La plupart des activités bancaires, et notamment les activités de marché, sont à forts rendements croissants, et donc sources de rentes et de distorsions de prix. Si l'on reste sur le terrain fiscal, la taxe sur les salaires aujourd'hui en vigueur peut être considérée comme une façon de taxer la rente. Dans Nicolaï et Schaff (2012), il est montré qu'elle joue peut-être même un rôle incitatif (en ayant un impact sur les rentes salariales). Elle pourrait être rendue plus progressive en fonction d'un facteur identifié comme lié à l'existence de rente : la taille du bilan par tête. La structure de taux d'imposition (elle-même déjà fonction des niveaux de salaire) serait plus ou moins élevée selon les banques, une année donnée, en fonction d'un indicateur de taille par tête. Cette taxe jouerait alors le double rôle de taxer la rente issue des rendements croissants et de réduire la profitabilité et l'incitation à trop user des avantages de cette structure de production. Si, comme le souligne Jean-François Lepetit, la taille du bilan n'est pas un bon indicateur car elle pénalise les activités pures de transformation, alors 
il est possible de considérer un indicateur du "levier à risque " : total du passif - fonds propres - dépôts, auquel nous préconisons d'ajouter une mesure de la taille du hors-bilan (engagements ou transactions, voir infra).

\section{Tableau}

Avantages et inconvénients des différentes stratégies fiscales

\begin{tabular}{|c|c|c|c|c|}
\hline & $\begin{array}{l}\text { Impact sur les } \\
\text { rentes des activités } \\
\text { de marché }\end{array}$ & $\begin{array}{l}\text { Limitation du } \\
\text { risque bancaire } \\
\text { systémique }\end{array}$ & $\begin{array}{c}\text { Frein à } \\
\text { l'accroissement } \\
\text { des possibles }\end{array}$ & $\begin{array}{l}\text { Faisabilité } \\
\text { technique }\end{array}$ \\
\hline $\begin{array}{l}\text { Taxe sur les salaires } \\
\text { avec différents taux } \\
\text { fonction d'un ratio } \\
\text { (engagements } \\
\text { sur effectifs) }\end{array}$ & $\begin{array}{l}\text { Oui, renforcé } \\
\text { par l'indexation } \\
\text { sur un élément } \\
\text { de « taille " }\end{array}$ & Faible* & Faible* & Simple \\
\hline $\begin{array}{l}\text { Taxe sur un agrégat } \\
\text { comptable intégrant la } \\
\text { valeur ajoutée ou PNB }\end{array}$ & $\begin{array}{l}\text { Idem, } \\
\text { en plus fort }\end{array}$ & Faible* & Faible* & Simple \\
\hline $\begin{array}{l}\text { Taxe sur les fonds } \\
\text { propres réglementaires }\end{array}$ & Oui & Oui & $\begin{array}{l}\text { Ambigu } \\
\text { (incitation à } \\
\text { l'innovation } \\
\text { financière) }\end{array}$ & Simple \\
\hline $\begin{array}{l}\text { Taxe actuelle sur } \\
\text { les transactions } \\
\text { financières }\end{array}$ & Faible & Faible & Faible & Simple \\
\hline $\begin{array}{l}\text { Taxe sur les } \\
\text { engagements associés } \\
\text { aux transactions } \\
\text { financières }\end{array}$ & Indirect, mais fort & Fort & Fort & Moins simple \\
\hline $\begin{array}{l}\text { Taxe sur les } \\
\text { engagements, calculés } \\
\text { sans compensations } \\
\text { algébriques }\end{array}$ & Indirect & Oui & Moyen & $\begin{array}{c}\text { Moyennement } \\
\text { complexe }\end{array}$ \\
\hline $\begin{array}{l}\text { Taxe sur les revenus et } \\
\text { les risques spécifiques } \\
\text { à chaque produit et } \\
\text { service commercialisés }\end{array}$ & Direct et fort & Moyen & Fort & $\begin{array}{c}\text { Complexe, } \\
\text { notamment } \\
\text { du fait de } \\
\text { la dimension } \\
\text { internationale }\end{array}$ \\
\hline
\end{tabular}

* Sauf si très forte progressivité et taux de la dernière tranche très élevé.

PNB : produit net bancaire.

Source : d'après les auteurs.

Dans cette logique, il serait d'ailleurs cohérent d'étendre la taxe sur les salaires à une taxe sur la valeur ajoutée - considérée ici comme un agrégat comptable (le produit net bancaire, PNB). Ainsi serait fiscalisé l'ensemble des facteurs (capital et travail) qui bénéficient de la rente. 


\section{Le risque systémique}

La taxe sur le risque systémique, mise en place à partir de 2011, a pour assiette les fonds propres réglementaires. C'est d'ailleurs la préconisation de Capelle-Blancard et Couppey-Soubeyran (2012).

L'argument est simple : les fonds propres réglementaires sont une mesure du risque pris par la banque. Ils résument efficacement l'ampleur des risques pris et, avec Bâle III, de mieux en mieux leur nature, y compris systémique.

Certes il convient de relativiser un tel optimisme : les fonds propres réglementaires sont une construction qui représente "au mieux » les engagements risqués des banques, mais le trajet est long entre la réalité opérationnelle et cette mesure synthétique (interviennent notamment de nombreux choix de modèles internes). De plus, il n'y a pas de comparabilité entre banques (des structures d'activité très différentes peuvent donner lieu au même niveau de fonds propres réglementaires). Enfin certains trouvent curieux de taxer ce qui ressemble déjà à un impôt (les fonds propres réglementaires sont un coût d'opportunité pour les actionnaires de la banque) : l'incitation à réduire au maximum ce qui doit être un instrument de pilotage des risques est surprenante. Les critiques en matière de gestion des risques bancaires sous Bâle II ont pour partie souligné une incitation à développer les modèles internes plus réglementaire que prudentielle.

L'un des arguments du rapport Lepetit contre l'assiette des fonds propres réglementaires était l'avantage que l'on donnait ainsi aux banques de marché vis-à-vis des banques universelles. L'évolution réglementaire a toutefois rééquilibré les impacts. Il n'en reste pas moins que l'activité de transformation demeure la principale contributrice des actifs pondérés du risque dans les grandes banques françaises.

Toutefois le choix des fonds propres réglementaires comme assiette se défend en pratique très bien : il s'agit là, d'une certaine façon, de la seule mesure de risque synthétique existante et, quoi qu'on en pense, les établissements bancaires cherchent déjà à les minimiser, indépendamment de toute taxe.

\section{Limiter l'accroissement des possibles}

Les nombreuses réflexions engagées à l'issue de la crise majeure que le monde connaît depuis 2007 incitent à orienter la fiscalité - notamment ici - en vue de décourager l'accroissement quasi infini des possibles que permet l'activité bancaire.

Chacun s'accorde pour différencier l'activité jugée " traditionnelle " de transformation au sens où nous l'avons définie supra et celle d'intermédiation purement financière. Nous avons toutefois noté que celle-ci 
est un levier pour celle-là. C'est donc l'ensemble de l'activité que l'on peut vouloir freiner ou limiter. L'objectif collectif serait alors de limiter l'accroissement des possibles, en considérant qu'au-delà d'une vitesse d'accroissement de ces possibles, notre système socioéconomique devient trop vulnérable. Nous examinons les différentes bases taxables sous ce rapport.

\section{Taxer la profitabilité?}

Il ne semble pas possible d'associer totalement innovation financière et profit comptable. La valorisation d'un produit dérivé se fait, comme nous l'avons indiqué, à la marge d'un bilan que l'on peut définir par son exposition à différents horizons temporels sur des facteurs de risque. Un produit peut "ne rien rapporter » en tant que tel et présenter une grande valeur car il " annule " des risques. Bien sûr, cette économie de risque se répercute en termes d'économie de fonds propres réglementaires, mais l'effet n'est pas forcément à la hauteur du gain procuré par l'innovation. De plus, l'incitation à réduire les fonds propres via l'ingénierie financière est en elle-même source de créativité !

Toutefois il est aujourd'hui techniquement possible - et les banques le font - de mesurer, lors de la création d'un nouveau produit, des éléments ex ante de rentabilité attendue et de risque, et ex post de mesurer les revenus engendrés. Ces calculs se font bien sûr sur la base de modèles et de données qui sont par définition imparfaits. La prise en considération du risque par ces données et modèles, aux côtés de la rentabilité, pourrait offrir une vraie incitation à ne pas s'aventurer dans des opérations où les risques sont mal maîtrisés et qui ne sont rentables que parce que le marché va dans une direction qui les valorise sur le moment. En allant à ce niveau fin de définition des assiettes taxables, on forcerait par ailleurs les banques à réconcilier plus systématiquement leurs évaluations ex ante et les mesures ex post des revenus et des pertes associés à la commercialisation d'un produit et service. Le rapport Lepetit renonçait à une telle approche en se fondant sur l'argument d'une comptabilité analytique insuffisamment sophistiquée. Cet argument est de moins en moins valide dans les grandes banques. La complexité requise par les nouvelles normes comptables et par les nouvelles règles prudentielles relativise l'effort à fournir pour atteindre ce degré de sophistication. Malheureusement, la mise en œuvre d'une telle fiscalité pose la question de la localisation des opérations et des bénéfices associés dans un environnement très international.

\section{Peut-on agir via les prix?}

Il n'est certes pas imaginable de définir des prix et des taxes sur ces prix pour des contrats de gré à gré aux formes si diverses. En revanche, 
il n'est pas absurde de taxer les prix sur les transactions des éléments constitutifs (obligations, actions, devises, matières premières supports à des marchés à terme, et même produits dérivés complexes standardisés). De par la nature d'un produit financier où le prix facial n'a pas de "sens ", une telle fiscalité revient à taxer les transactions.

La taxe sur les transactions financières mise en place en France est cantonnée aux échanges de produits simples comme les actions ${ }^{7}$. Le projet de directive européenne (Conseil européen, 2011), en revanche, visait également les transactions sur produits dérivés. Dans ce cas, la valeur notionnelle des contrats est retenue comme assiette à la taxe. La directive européenne n'a pas vu le jour jusqu'ici. La France semblait souhaiter relancer les discussions, mais la situation liée au Brexit risque de réduire l'ambition de cette taxe à celle existante aujourd'hui en France.

\section{Peut-on taxer les encours?}

Deux façons d'apporter des réponses, au travers de deux questions :

- ne faut-il pas taxer l'activité centrale, celle de transformation, lorsqu'elle dépasse un certain niveau, ce dernier témoignant implicitement de l'existence d'un levier ? On limiterait alors directement l'accroissement des possibles dans l'économie réelle et on réduirait l'incitation et le support au développement d'un levier ;

- comment intégrer le dimensionnement de l'activité hors-bilan, en vue de réduire la création de possibles pour l'activité de transformation, au travers du levier que présentent les activités d'intermédiation ?

Si la première question est peu dans l'air du temps, elle mérite réflexion quand on se souvient de l'origine de la crise sur la dette européenne : c'est le support de marché le plus basique (obligations d'État) qui est à l'origine des spirales enchevêtrées des deux sphères financières et réelles en Europe. Notons également que le rapport Liikanen (2012) invite la Commission européenne à se pencher sur la question des prêts immobiliers à l'origine de la crise des subprimes.

Concernant la seconde question, le montant total des engagements est une mesure de cette activité hors-bilan - ce que n'offre pas une mesure de leur juste valeur. Il convient également de raisonner en l'absence de compensation - certes naturelle en termes de mesure du risque financier, mais moins en termes d'activité - entre des positions à différents horizons ou vis-à-vis de différents tiers.

La mesure de cette activité hors-bilan par l'engagement hors-bilan global (compensé, donc) ou par leur juste valeur inscrite au bilan constitue des indications des risques prudentiels et non d'activité ; dès lors, elle ne permet pas de limiter l'innovation financière, puisque, à l'inverse, elle l'incite : pour se défaire de structures de risques résiduels, 
l'ingénierie financière va "créer " un nouveau produit : il n'y aura pas incitation à prendre moins de risque en créant moins de produits, mais bien au contraire incitation à créer de nouveaux produits pour réduire les risques liés aux produits déjà en portefeuille.

Il semble donc nécessaire de considérer (aux côtés des éléments bilanciels et prudentiels) les «engagements bruts hors-bilan» pour disposer d'une assiette qui rende réellement compte de l'activité de levier des banques.

\section{Prise en compte du hors-bilan}

Rappelons que nous appelons hors-bilan essentiellement les produits dérivés aujourd'hui inscrits au bilan dans le portefeuille de transaction. $\mathrm{Au}$ total, ce hors-bilan pourrait être pris en considération dans la fiscalité, à deux niveaux :

- les effets « taille" : le hors-bilan doit être associé au « dimensionnement » de la taxe sur les salaires. Nous préconisons un ratio du type (engagements bilan ${ }^{8}+$ engagements hors-bilan) sur effectifs ;

- les effets « levier » qui sont soupçonnés d'engendrer un trop grand accroissement des possibles : ici il est tenu compte du seul hors-bilan, puisque c'est l'un des facteurs de la "fonction de production" qui " produit " le transfert de risque. Nous préconisons une taxe assise sur les engagements associés aux transactions financières.

Deux remarques peuvent alors être faites. En premier lieu, mesurer la «taille» par une photo de fin d'exercice ou de fin de trimestre ne retrace pas complètement l'activité au cours de la période, comme les récentes déclarations du Comité de Bâle que nous rappelions en introduction le soulignent. Or il semble que ce soit ce flux d'activités qui fasse apparaitre, d'une part, les effets d'échelle et, d'autre part, les activités de recherche de valeur au travers d'un transfert incomplet des risques. En second lieu, les transactions telles qu'envisagées par la taxe sur les transactions financières doivent correctement prendre en compte les instruments dérivés, contrairement à ce qui est le cas dans sa déclinaison actuelle en France. Le projet de directive européenne stipulait que l'assiette taxable doit être le "notionnel " des contrats, tout en précisant qu'il conviendra de veiller à ce qu'il n'y ait pas de contournement. Nous pensons que la mesure des engagements associés est une base taxable plus pertinente : elle correspond généralement au " notionnel ", mais a l'avantage d'être indépendante de la façon dont est rédigé le contrat et structuré le produit ; surtout elle oblige, au niveau d'un produit complexe, à identifier les différents engagements induits et non un montant global notionnel qui compense et rend invisibles les multiples engagements. Tout actif complexe devrait donc être "déconstruit " pour additionner les différents engagements hors-bilan 
qu'il implique et l'assiette de la taxe sur les transactions en matière de produits dérivés serait l'engagement afférent. Dans un tel cadre, la "fonction de production" qui consiste à gérer des couvertures de manière dynamique est effectivement freinée et de nombreux produits complexes perdent leur intérêt économique, ce qui correspond bien à l'objectif initial de réduction de l'activité financière.

Ainsi, tant pour la fiscalité ciblée sur la rente du système bancaire que pour celle qui cible l'effet de levier qu'apporte le système bancaire, le plus simple est - pour mesurer l'effet " taille " comme l'effet " activité ou levier »- de considérer la taille du bilan en termes d'engagement, à laquelle on ajoute une mesure des engagements hors-bilan, " en valeur absolue ", non en valeur algébrique et non pondérée par une actualisation.

Pour ce qui relève de la taxation de l'activité elle-même, les taux de taxation ne peuvent plus être alors du même montant que ceux prévus, par exemple, aujourd'hui par la taxe sur les transactions financières. Même pour des taux de taxation bien plus faibles, la pénalisation des activités sera effective car, comme nous l'avons montré, elle interviendra dans le processus même de production.

\section{CONCLUSION}

Finalement, qu'est-ce qui différencie le secteur financier des autres secteurs économiques?

La création de possibles par l'innovation, les rendements d'échelle, la prise de risque engageant l'ensemble de l'économie sont des éléments que l'on retrouve dans de nombreux autres secteurs d'activité (développement de centrales nucléaires, pour prendre un exemple extrême).

Pour résumer le fond de notre pensée, il nous semble que la forme de rendements d'échelle dans la finance est sans réelle limite - comme en témoigne l'hyperinflation des bilans et des hors-bilans bancaires à laquelle nous avons assisté jusqu'à la crise. Par ailleurs, la décentralisation de décisions qui se répercute d'une manière non marginale sur les comptes d'une banque donne au court terme un poids très important. La convergence d'intérêts entre des actionnaires à l'horizon trop court, des opérateurs et des clients pour " créer " des produits qui présentent des risques extrêmes n'existe pas dans d'autres environnements (le nucléaire est très régulé souvent par un actionnaire public, les clients n'ont qu'un intérêt marginal à la prise de risque de l'opérateur, etc.). Surtout, aujourd'hui, la fonction de contrôle dans les établissements bancaires vérifie certes que les processus sont correctement respectés, mais ces processus visent justement à créer des risques extrêmes. Enfin l'interconnexion des marchés, la masse des 
dettes publiques, etc. dessinent un terrain de jeu d'une taille démesurée sur laquelle tout levier engendre des chiffres astronomiques (primes, profits, échanges, risques).

Nous en déduisons trois recommandations :

- une taxation des rentes du secteur bancaire peut se faire via une taxe sur les salaires, avec une différenciation des taux d'imposition fonction d'un ratio du type total des engagements (bilan et hors-bilan) sur effectifs. Il est possible d'aller plus loin et de considérer l'agrégat comptable valeur ajoutée ou PNB plutôt que les salaires, si l'on renonce à différencier les salaires par niveaux ;

- une taxation du risque systémique bancaire peut être assise sur les fonds propres réglementaires (abrogée en 2019 dans le cas français ${ }^{9}$ ) ; - une taxation visant à "maîtriser l'accroissement des possibles" peut être construite via une taxe assise sur les engagements associés aux transactions financières. Une assiette en termes d'encours (engagements bilan et hors-bilan non consolidés) est également possible. Une mesure plus individualisée lors de la création de nouveaux produits financiers serait idéale et serait rapidement envisageable, compte tenu de la sophistication accélérée des comptabilités analytiques des banques.

\section{NOTES}

1. Voir Nicolaï et Schaff (2012).

2. La BCE (Banque centrale européenne) via le Mécanisme de supervision unique (MSU) supervise aujourd'hui les banques européennes les plus importantes et le Mécanisme de résolution unique (MRU) permet, en cas de défaut d'une banque, de maîtriser la contribution des citoyens (via un renflouement par les États) en mettant préférentiellement à contribution les actionnaires et les créanciers. En revanche, des limites importantes subsistent et le projet de Système européen d'assurance des dépôts (SEAD) n'est toujours pas adopté, certains États refusant toujours de mutualiser ces risques entre pays membres.

3. Le hors-bilan des banques tel qu'il était jusque dans les années 1990 n’existe plus. Les produits dérivés notamment ont été réintégrés dans le bilan "à la juste valeur " dans les nouvelles normes comptables IFRS. Dans cet article néanmoins, nous utiliserons ce terme pour mieux rendre compte du rôle que jouent les produits dérivés du portefeuille de transaction (cf. infra).

4. Voir le site : https://www.bis.org/publ/bcbs_nl20_fr.htm.

5. À noter que cette norme concernant la juste valeur, appelée IAS 39, est aujourd'hui amendée par la nouvelle règle IFRS 9 qui permet de revenir à des pratiques de macrocouverture. IAS 39 avait interdit de couvrir (et donc de faire disparaitre comptablement) le risque d'un portefeuille d'instruments au travers d'une seule couverture (justifiée par les corrélations entre instruments au sein du portefeuille, mais dangereuse du fait d'une instabilité de ces corrélations en cas de crise). IFRS 9 rouvre la porte à une telle pratique depuis cette année.

6. À noter que la banque utilise la stabilité statistique de ses ressources pour également jouer un rôle d'investisseur en fonds propres des entreprises et pas seulement en fonds investis sur des titres de dette ou de crédit. Des organismes financiers comme les OPCVM tiennent le même rôle de transformation par diversification et professionnalisation que la banque, mais du côté de l'épargne. Les compagnies 
d'assurance-vie font de même. Dans les deux cas, l'épargne des agents économiques fournit un passif plus ou moins diversifié qui, grâce à ces deux intermédiaires (OPCVM et assurance-vie), peut s'investir en bénéficiant d'un accès à des risques théoriquement diversifiés à un coût faible. Cette collecte d'épargne permet le financement de l'économie, au même titre que les banques.

7. Il existe également une taxe visant le "trading à haute fréquence ". Cette activité est aujourd'hui montrée du doigt parce qu'elle représente des volumes de transactions très importants. Elle ne semble toutefois pas avoir joué de rôle majeur dans la/les crise(s) depuis 2007. En revanche, elle et sans doute un lieu de rente élevée.

8. Soit le total du bilan, moins les fonds propres, moins les dépôts, de façon à ne pas pénaliser les banques de dépôts, comme le recommande le rapport Lepetit (2010).

9. Elle est progressivement remplacée par une taxe pour le financement du fonds de soutien aux collectivités territoriales ayant souscrit un "emprunt toxique».

\section{BIBLIOGRAPHIE}

Braudel F. (1979), Civilisation matérielle, économie et capitalisme - 2. Les jeux de l'échange, Armand Colin.

Capelle-Blancard G. et Couppey-Soubeyran J. (2012), La taxation des activités bancaires sous l'angle de la régulation financière, Conseil des prélèvements obligatoires, Rapport particulier $\mathrm{n}^{\circ} 4$, septembre.

Conseil européen (2011), Directive on a Common System of Financial Transaction Tax and Amending Directive, 2008/7/EC, COM(2011) 594 final (2011).

Conseil européen (2015), Proposition de règlement du Parlement européen et du Conseil relatifà des mesures structurelles améliorant la résilience des établissements de crédit de l'UE - Orientation générale, 10150/15 COR 1, EF 121, ECOFIN 528 CODEC 910.

CPO (Conseil des prélèvements obligatoires) (2013), Les prélèvements obligatoires et les entreprises du secteur financier, 24 janvier.

LePETIT J.-F. (2010), Rapport sur le risque systémique, à la demande du ministre de l'Économie et des Finances Christine Lagarde.

LiIKANEN E. (2012), Rapport de la Commission d'experts européen sur la réforme bancaire, octobre.

Nicolaï J.-P. et SCHAfF C. (2012), Salaires et emploi dans la finance, Conseil des prélèvements obligatoires, complément au rapport particulier $\mathrm{n}^{\circ} 4$, annexe du rapport Les prélèvements obligatoires sur les entreprises du secteur financier, septembre.

Pascali L. (2016), "Banks and Development: Jewish Communities in the Italian Renaissance and Current Economic Performance ", The Review of Economics and Statistics, vol. 98, n 1, pp. 140-158. 
